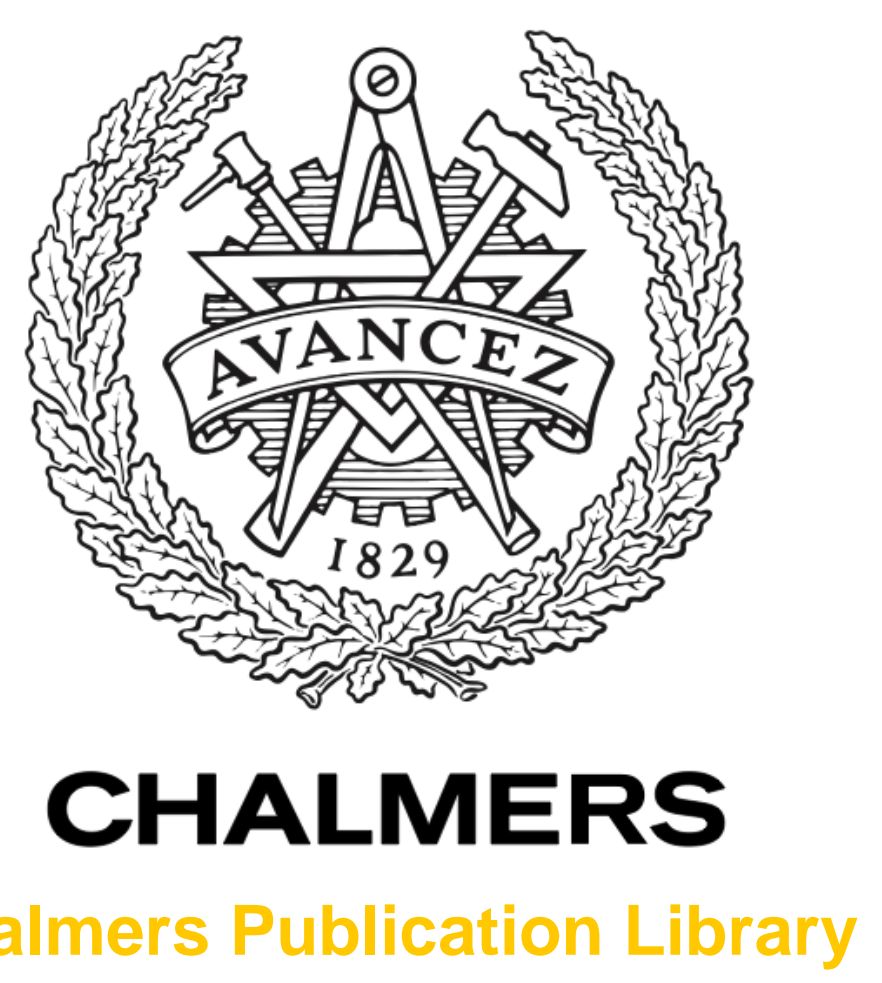

Chalmers Publication Library

\title{
Variational Bayesian EM for SLAM
}

This document has been downloaded from Chalmers Publication Library (CPL). It is the author's version of a work that was accepted for publication in:

IEEE 6th International Workshop on Computational Advances in Multi-Sensor Adaptive Processing (CAMSAP), 2015, Cancun, Mexico, 13-16 Dec. 2015

Citation for the published paper:

Fatemi, M. ; Svensson, L. ; Hammarstrand, L. et al. (2015) "Variational Bayesian EM for SLAM". IEEE 6th International Workshop on Computational Advances in Multi-Sensor Adaptive Processing (CAMSAP), 2015, Cancun, Mexico, 13-16 Dec. 2015 pp. 501-504.

http://dx.doi.org/10.1109/CAMSAP.2015.7383846

Downloaded from: http://publications.lib.chalmers.se/publication/231151

Notice: Changes introduced as a result of publishing processes such as copy-editing and formatting may not be reflected in this document. For a definitive version of this work, please refer to the published source. Please note that access to the published version might require a subscription.

Chalmers Publication Library (CPL) offers the possibility of retrieving research publications produced at Chalmers University of Technology. It covers all types of publications: articles, dissertations, licentiate theses, masters theses, conference papers, reports etc. Since 2006 it is the official tool for Chalmers official publication statistics. To ensure that Chalmers research results are disseminated as widely as possible, an Open Access Policy has been adopted.

The CPL service is administrated and maintained by Chalmers Library. 


\title{
Variational Bayesian EM for SLAM
}

\author{
Maryam Fatemi, Lennart Svensson, Lars Hammarstrand and Malin Lundgren \\ Signals and Systems \\ Chalmers University of Technology \\ Gothenburg, Sweden \\ Email: \{maryam.fatemi,lennart.svensson,lars.hammarstrand,malin.lundgren\}@ chalmers.se
}

\begin{abstract}
Designing accurate, robust and cost-effective systems is an important aspect of the research on self-driving vehicles. Radar is a common part of many existing automotive solutions and it is robust to adverse weather and lighting conditions, as such it can play an important role in the design of a self-driving vehicle. In this paper, a radar-based simultaneous localization and mapping (SLAM) algorithm using variational Bayesian expectation maximization (VBEM) is presented. The VBEM translates the inference problem to an optimization one. It provides an efficient and powerful method to estimate the unknown data association variables as well as the map of the environment as perceived by a radar and the unknown trajectory of the vehicle.
\end{abstract}

\section{INTRODUCTION}

During recent years, self-driving cars have been the subject of extensive research. Many different functionalities expected from a self-driving vehicle are facilitated by having an accurate map of the environment and the ability to localize accurately within that map. The problem of mapping an unknown environment and estimating the unknown trajectory of the vehicle concurrently, is referred to as simultaneous localization and mapping (SLAM) [1] [2].

For designing a robust SLAM solution in a cost-effective manner, it is important to explore the use of different sensors as well as different combinations of sensors to achieve the desired functionalities. In [3] the authors present a SLAM solution using a high-end Velodyne (64-beam rotating Lidar) sensor. Another SLAM solution using the Velodyne sensor is described in [4]. In [5], the authors present a vision based SLAM. Although high-end sensors such as the Velodyne can provide accurate solutions, the price of such sensors and the manufacturers' preference to avoid altering the appearance of their vehicles by mounting large sensors on them, have led researchers to look into more cost-effective solutions. For instance, [6] presents an example of autonomous driving using close-to-the-market sensors. Size and cost of the camera, among other things, make it a desirable sensor for automotive applications. However, the camera is strongly affected by adverse weather and ambient lighting conditions.

Radar is an important sensor that is commonly used in different automotive applications. Radar provides the relative range, angle and speed to objects detected in its field of view. Therefore, radar detections can be used to build a map of the static as well as the dynamic objects of the environment. In [7], authors compare a radar to a laser range finder and conclude that the radar is less susceptible to occlusions and can penetrate further into the environment. In addition compared to other range finders, a radar's performance is less affected by dust, smoke, undesirable weather conditions and ambient light situation [7]. Furthermore, the size of automotive radars are such that they can easily be fitted into cars. Consequently, a radar-based SLAM solution seems appealing, if not as a standalone sensor at least as an important component of a robust, accurate and cost-effective solution.

Construction of radar maps are discussed in [7] [8] where the probability hypothesis density (PHD) filter is used to estimate a map of the environment. In addition, radar-based SLAM solutions using the PHD filter are presented in [9] [10]. In most of these methods the landmarks are modelled as point targets. This assumption is not completely accurate for landmarks whose size is not negligible compared to their distance to the sensor [11]. Such landmarks can be more accurately modelled as extended objects [12]. In [13] a radar map is built assuming known trajectory of the sensor and using expectation maximization (EM) [14] and VBEM [15] methods. The VBEM translates an inference problem to an optimization one and it is guaranteed to find a local optimum [15]. This method is computationally efficient and easy to implement. Furthermore, the VBEM can deal with unknown number of landmarks and can tackle the uncertainties in the data associations efficiently.

In this paper, we extend on the ideas in [13] and develop a radar-based SLAM solution using VBEM. In this algorithm the joint posterior density of the map, the poses and the data associations is approximated as a product of three variational distributions. These distributions are found using the VBEM method. From the estimated joint posterior density, the parameters of the map, the data association probabilities and the poses are inferred. The presented solution is a batch algorithm.

\section{Problem Formulation}

The overall objective is to solve a SLAM problem, that is, to jointly estimate a map of the environment and the vehicle trajectory. The map is assumed to be static and is primarily made up of a set of landmarks that have unknown extensions, locations and expected number of detections; the map also describes the distribution over the clutter measurements. The vehicle is assumed to follow an unknown trajectory and collect radar measurements at every time instant. We intend to solve the SLAM problem, by computing the posterior density of the vehicle trajectory, the map and the data associations, 
i.e., $p\left(\mathbf{X}_{1: M}, \mathbf{Z}_{1: M}, \theta \mid \mathbf{Y}_{1: M}\right)$ where $\mathbf{X}_{1: M}, \mathbf{Z}_{1: M}$ and $\mathbf{Y}_{1: M}$ denote the poses of the vehicle, the data associations and the measurements from time 1 to $\mathrm{M}$, respectively, and $\theta$ contains all the parameters that describe the map.

The trajectory of the vehicle is described by its pose at each time instant. The pose at time $m$ consists of the position of the vehicle $\mathbf{X}_{m}^{p}$, in the global Cartesian coordinate system and its heading $X_{m}^{\phi}$. The movement of the vehicle is expressed as

$$
\mathbf{X}_{m}=\mathbf{X}_{m-1}+\mathbf{u}_{m-1}+\mathbf{v}_{m}
$$

where $\mathbf{X}_{m}$ denotes the vehicle's pose at time $m, \mathbf{u}_{m-1}$ is the odometry and $\mathbf{v}_{m} \sim \mathcal{N}\left(\mathbf{0}, \mathbf{Q}_{m}\right)$ is the odometry noise.

The measurements are assumed to be generated by an inhomogeneous Poisson process. As a result, the number of detections at each time instant follows a Poisson distribution and the location of the measurements is described by an intensity function over the observed space. The intensity function describes the spatial distribution of the map and is modelled by a constant clutter level plus a Gaussian mixture. Each component of this mixture is referred to as a landmark. Consequently, the map is denoted by $\theta=\left[\mu_{1: K}, \boldsymbol{\Sigma}_{1: K}, \omega_{1: K}, \lambda_{c}\right]$ where $\mu_{j}=\left[\mu_{j}^{x}, \mu_{j}^{y}\right], \Sigma_{j}$ and $\omega_{j}$ denote the location of each landmark in the global Cartesian coordinate system, the spread of the detections generated by each landmark (the extension of the landmark) and the expected number of detections from each landmark, respectively. Additionally, the expected number of clutter detections is denoted by $\lambda_{c}$ and $K$ is the number of landmarks. Assuming that the $i$ th measurement at time $m$ is generated by landmark $j$, the measurement equation is expressed as

$$
\mathbf{y}_{m}^{i}=\left[\begin{array}{c}
\left\|\mu_{j}+\mathbf{d}_{j}-\mathbf{X}_{m}^{p}\right\| \\
\angle \mu_{j}+\mathbf{d}_{j}-\mathbf{X}_{m}^{p}-\mathbf{X}_{m}^{\phi}
\end{array}\right]+\mathbf{w}_{m}
$$

where $\mathbf{d}_{j} \sim \mathcal{N}\left(\mathbf{0}, \boldsymbol{\Sigma}_{j}\right)$ describes the extension of the landmark and $\mathbf{w}_{m} \sim \mathcal{N}(\mathbf{0}, \mathbf{R})$ is the measurement noise.

The field of view, denoted by $f\left(\mu_{j}, \mathbf{X}_{m}\right)$, is a function of the vehicle's pose and the location of the landmark. We assume that the associations between landmarks and measurements are unknown. Consequently, the source of each measurement could either be clutter or any of the landmarks in the field of view of the sensor. In this paper, the data association variables are modelled as binary random variables. Accordingly, $z_{m}^{i j}$ denotes the data association between measurement $i$ and landmark $j$ at time $m$ and is equal to one if the measurement is generated by this landmark and zero otherwise.

\section{VBEM-SLAM}

This paper presents a solution to the SLAM problem based on the VBEM. The objective is to estimate the posterior density $p\left(\mathbf{X}_{1: M}, \mathbf{Z}_{1: M}, \theta \mid \mathbf{Y}_{1: M}\right)$, but since it is intractable to calculate this density exactly, resorting to approximations is inevitable. In the VBEM framework, the posterior density is approximated by a simpler distribution that assumes certain independencies. The VBEM method optimizes this distribution so that it is closest to the true posterior in the "exclusive"
Kullback-Liebler divergence sense [16]. This is equivalent to maximizing a lower bound on the log marginal likelihood [15].

In this paper, the joint posterior density is approximated by

$p\left(\mathbf{X}_{1: M}, \mathbf{Z}_{1: M}, \theta, \mathbf{Y}_{1: M}\right) \approx q_{z}\left(\mathbf{Z}_{1: M}\right) q_{\Sigma}\left(\boldsymbol{\Sigma}_{1: K}\right) q_{X, \theta^{\prime}}\left(\mathbf{X}_{1: M}, \theta^{\prime}\right)$,

where $\theta^{\prime}=\left[\mu_{1: K}, \omega_{1: K}, \lambda_{c}\right]$. In this factorization, the data associations are considered to be independent from the map and the poses. This factorization enables us to search for an optimal posterior density in the KLD sense. More specifically, we can use a coordinate descent algorithm to analytically solve for each variational distribution given the other two. The solution to the minimization of the "exclusive" KLD between the two densities in (3) is

$$
\begin{gathered}
q_{z}\left(\mathbf{Z}_{1: M}\right) \propto \exp \left(\mathbb{E}_{q_{\Sigma}, q_{X, \theta^{\prime}}}\left\{\log p\left(\mathbf{X}_{1: M}, \mathbf{Z}_{1: M}, \theta, \mathbf{Y}_{1: M}\right)\right\}\right) \\
q_{\Sigma}\left(\boldsymbol{\Sigma}_{1: K}\right) \propto \exp \left(\mathbb{E}_{q_{Z}, q_{X, \theta^{\prime}}}\left\{\log p\left(\mathbf{X}_{1: M}, \mathbf{Z}_{1: M}, \theta, \mathbf{Y}_{1: M}\right)\right\}\right)
\end{gathered}
$$

$q_{X, \theta^{\prime}}\left(\mathbf{X}_{1: M}, \theta^{\prime}\right) \propto \exp \left(\mathbb{E}_{q_{\Sigma}, q_{Z}}\left\{\log p\left(\mathbf{X}_{1: M}, \mathbf{Z}_{1: M}, \theta, \mathbf{Y}_{1: M}\right)\right\}\right)$

\section{A. Derivation of the joint density}

The purpose is to derive the necessary expressions required for implementing (4)-(6). We begin by expressing the joint distribution of all variables as a product of two slightly simpler distributions,

$p\left(\mathbf{X}_{1: M}, \mathbf{Z}_{1: M}, \theta, \mathbf{Y}_{1: M}\right)=p\left(\mathbf{Y}_{1: M}, \mathbf{Z}_{1: M} \mid \mathbf{X}_{1: M}, \theta\right) p\left(\mathbf{X}_{1: M}, \theta\right)$.

Given that the measurements are collected at $M$ independent time scans, the complete data likelihood can be factorized as

$$
p\left(\mathbf{Y}_{1: M}, \mathbf{Z}_{1: M} \mid \mathbf{X}_{1: M}, \theta\right)=\prod_{m=1}^{M} p\left(\mathbf{Y}_{m}, \mathbf{Z}_{m} \mid \mathbf{X}_{m}, \theta\right)
$$

where each factor can be written as

$$
p\left(\mathbf{Y}_{m}, \mathbf{Z}_{m} \mid \mathbf{X}_{m}, \theta\right)=p\left(\mathbf{Y}_{m} \mid \mathbf{Z}_{m}, \mathbf{X}_{m}, \theta\right) \operatorname{Pr}\left\{\mathbf{Z}_{m} \mid \mathbf{X}_{m}, \theta\right\} .
$$

Using (2), the likelihood is expressed as

$$
p\left(\mathbf{Y}_{m} \mid \mathbf{Z}_{m}, \mathbf{X}_{m}, \theta\right)=\prod_{i=1}^{n_{m}} \prod_{j=1}^{K}\left(p\left(\mathbf{y}_{m}^{i} \mid \mathbf{Z}_{m}, \mathbf{X}_{m}, \theta\right)\right)^{z_{m}^{i j}}\left(\frac{1}{V}\right)^{z_{m}^{i 0}}
$$

where $n_{m}$ is the number of measurements at time $m, V$ is the sensor observation volume and $z_{m}^{i 0}$ denotes the clutter data association. The probability mass function of the data associations at each time instant can be written as

$$
\begin{aligned}
\operatorname{Pr}\left\{\mathbf{Z}_{m} \mid \mathbf{X}_{m}, \theta\right\} & =\operatorname{Pr}\left\{\mathbf{Z}_{m}, n_{m} \mid \mathbf{X}_{m}, \theta\right\} \\
& =\operatorname{Pr}\left\{\mathbf{Z}_{m} \mid n_{m}, \mathbf{X}_{m}, \theta\right\} \operatorname{Pr}\left\{n_{m} \mid \mathbf{X}_{m}, \theta\right\},
\end{aligned}
$$

where the probability of receiving $n_{m}$ measurements at time $m$ follows a Poisson distribution

$$
\operatorname{Pr}\left\{n_{m} \mid \mathbf{X}_{m}, \theta\right\}=\lambda_{m}^{n_{m}} \frac{e^{-\lambda_{m}}}{n_{m} !}
$$


with the intensity of $\lambda_{m}=\lambda_{c}+\sum_{r} f\left(\mu_{r}, \mathbf{X}_{m}\right) \omega_{r}$. Moreover,

$$
\operatorname{Pr}\left\{\mathbf{Z}_{m} \mid n_{m}, \mathbf{X}_{m}, \theta\right\}=\prod_{i=1}^{n_{m}}\left(\frac{\lambda_{c}}{\lambda_{m}}\right)^{z_{m}^{i 0}} \prod_{j=1}^{K}\left(\frac{\omega_{j} f\left(\mu_{j}, \mathbf{X}_{m}\right)}{\lambda_{m}}\right)^{z_{m}^{i j}}
$$

To complete the joint density, the prior density of the vehicle trajectory and the map must be defined. Using (1) the prior density of the vehicle's trajectory is expressed as $p\left(\mathbf{X}_{1: M}\right)=\prod_{m=1}^{M} p\left(\mathbf{X}_{m} \mid \mathbf{X}_{m-1}\right)$. Additionally, assuming that the map parameters are independent prior to making observations, the prior density of the map is described by $p(\theta)=p\left(\lambda_{c}\right) \prod_{j=1}^{K} p\left(\mu_{j}\right) p\left(\boldsymbol{\Sigma}_{j}\right) p\left(\omega_{j}\right)$, where we assume that

$$
\begin{gathered}
p(\theta)=\mathcal{G} \mathcal{A} \mathcal{M}\left(\lambda_{c} ; c^{0}, e^{0}\right) \prod_{j=1}^{K} \mathcal{N}\left(\mu_{j} ; \bar{\mu}_{j}^{0}, \mathbf{P}_{j}^{0}\right) \\
\mathcal{I} \mathcal{W}\left(\boldsymbol{\Sigma}_{j} ; \mathbf{S}_{j}^{0}, \nu_{j}^{0}\right) \mathcal{G} \mathcal{A} \mathcal{M}\left(\omega_{j} ; a_{j}^{0}, b_{j}^{0}\right) .
\end{gathered}
$$

$\mathcal{G} \mathcal{A M}(\omega ; a, b)$ denotes a gamma distribution with parameters $a$ and $b$, and $\mathcal{I} \mathcal{W}(\boldsymbol{\Sigma} ; \mathbf{S}, \nu)$ denotes an inverse Wishart distribution with parameters $\mathbf{S}$ and $\nu$. The prior density of the extensions is assumed to be Inverse Wishart. This density is conjugate to a Gaussian likelihood, as such, it provides us with a tractable solution for estimating the extension of the objects. The prior density of the weights and the expected number of clutter measurements are assumed to follow the Gamma distribution since this density is conjugate to the Poisson distribution of the expected number of measurements.

\section{B. Simplifying Assumptions and Approximations}

An important property of the VBEM algorithm is that it provides tractable solutions for models belonging to the conjugate exponential (CE) family [17]. As such, in this paper the following simplifying assumptions and approximations are made so that the model remains in the CE family.

First, (2) is linearised using first order Taylor expansion. The resulting measurement model is

$$
\begin{aligned}
\mathbf{y}_{m}^{i} & =\mathbf{H}_{x \mu}\left[\begin{array}{c}
\mathbf{X}_{m} \\
\mu_{j}
\end{array}\right]+\delta_{m}^{i j}+\mathbf{H}_{d} \mathbf{d}_{j}+\mathbf{w}_{m} \\
& =\mathbf{H}_{x \mu}\left[\begin{array}{c}
\mathbf{X}_{m} \\
\mu_{j}
\end{array}\right]+\delta_{m}^{i j}+\mathbf{w}_{m}^{\prime}
\end{aligned}
$$

where $\mathbf{H}_{x \mu}$ and $\mathbf{H}_{d}$ are Jacobians of the measurement model w.r.t $\mathbf{X}_{m}, \mu_{j}$ and $\mathbf{d}_{j}$, respectively. $\delta_{m}^{i j}$ is a constant resulting from the linearisation and $\mathbf{w}^{\prime}{ }_{m} \sim \mathcal{N}(\mathbf{0}, \tilde{\mathbf{R}})$, where $\tilde{\mathbf{R}}=\mathbf{R}+$ $\mathbf{H}_{d} \boldsymbol{\Sigma}_{j} \mathbf{H}_{d}^{T}$. In this paper, it is assumed that $\tilde{\mathbf{R}} \approx \mathbf{H}_{d} \boldsymbol{\Sigma}_{j} \mathbf{H}_{d}^{T}$, i.e., the measurement noise is negligible compared to the extension of the landmarks. This assumption allows us to maintain an inverse Wishart distribution over the extensions.

Second, in each iteration of the algorithm, the field of view function is approximated using the best available guess about the vehicles's pose and the position of the landmarks. More specifically, at iteration $\mathrm{t}+1$, the field of view function is approximated by $f\left(\mathbf{X}_{m}, \mu_{j}\right) \approx f\left(\hat{\mathbf{X}}_{m}^{t}, \hat{\mu}_{j}^{t}\right)$. Moreover, this function is equal to one if landmark $j$ is in the field of view of the vehicle at pose $\mathbf{X}_{m}$ and is equal to zero otherwise.

\section{The VBEM-SLAM algorithm}

In this algorithm, each iteration consists of updating $q_{z}\left(\mathbf{Z}_{1: M}\right), q_{\Sigma}\left(\boldsymbol{\Sigma}_{1: K}\right)$, and $q_{X, \theta^{\prime}}\left(\mathbf{X}_{1: M}, \theta^{\prime}\right)$ according to Equations (4), (5) and (6), respectively. In order to perform these calculations, it is assumed that the variational distributions of the parameters of the map and the poses have the same type as the one in the joint distribution. That is,

$$
\begin{gathered}
q_{\Sigma}\left(\boldsymbol{\Sigma}_{1: K}\right)=\prod_{j=1}^{K} \mathcal{I} \mathcal{W}\left(\boldsymbol{\Sigma}_{j} ; \mathbf{S}_{j}^{q}, \nu_{j}^{q}\right) \\
q_{X, \theta^{\prime}}\left(\mathbf{X}_{1: M}, \theta^{\prime}\right)=\mathcal{N}\left(\left[\mathbf{X}_{1: M}^{T}, \mu_{1: K}^{T}\right]^{T} ;\left[\overline{\mathbf{X}}_{1: M}^{q, T}, \bar{\mu}_{1: M}^{q, T}\right]^{T}, \mathbf{W}^{\mathrm{q}}\right) \\
\mathcal{G} \mathcal{A} \mathcal{M}\left(\omega_{j}, a_{j}^{q}, b_{j}^{q}\right) \mathcal{G} \mathcal{A} \mathcal{M}\left(\lambda_{c}, c^{q}, e^{q}\right) .
\end{gathered}
$$

Furthermore, the variational distribution of the data associations has the following form

$$
q_{z}\left(\mathbf{Z}_{1: M}\right)=\prod_{m=1}^{M} \prod_{i=1}^{n_{m}}\left(\pi_{m}^{i 0}\right)^{z_{m}^{i 0}} \prod_{j=1}^{K}\left(\pi_{m}^{i j}\right)^{z_{m}^{i j}},
$$

where $\pi_{m}^{i j}$ is the probability that measurement $\mathbf{y}_{m}^{i}$ is generated by landmark $j$ and $\pi_{m}^{i 0}$ is the probability that measurement $\mathbf{y}_{m}^{i}$ is clutter. Since each measurement can be generated by either of the landmarks or is clutter, $\pi_{m}^{i 0}+\sum_{j=1}^{K} \pi_{m}^{i j}=1$. These probabilities are calculated using (4), (11) and (12), according to a similar procedure as the one explained in [13].

Similarly, using (5), (12) and (13), the parameters of $q_{\Sigma}\left(\boldsymbol{\Sigma}_{1: K}\right)$ are updated as

$$
\begin{aligned}
& \nu_{j}^{q}=\nu_{j}^{0}+\sum_{m=1}^{M} \sum_{i=1}^{n_{M}} \mathbb{E}_{q_{\mathbf{Z}}}\left\{z_{m}^{i j}\right\} \\
& \mathbf{S}_{j}^{q}=\mathbf{S}_{j}^{0}+\sum_{m=1}^{M} \sum_{i=1}^{n_{M}} \mathbb{E}_{q \mathbf{Z}}\left\{z_{m}^{i j}\right\}\left(\mathbf{H}_{d}^{T}\right)^{-1} \\
& \quad\left(\left(\mathbf{y}_{m}^{i}-\mathbf{H}_{x \mu}\left[\begin{array}{c}
\overline{\mathbf{X}}_{m}^{q} \\
\bar{\mu}_{j}^{q}
\end{array}\right]-\delta_{m}^{i j}\right)\left(\mathbf{y}_{m}^{i}-\mathbf{H}_{x \mu}\left[\begin{array}{c}
\overline{\mathbf{X}}_{m}^{q} \\
\bar{\mu}_{j}^{q}
\end{array}\right]-\delta_{m}^{i j}\right)^{T}\right. \\
& \left.\quad+\mathbf{H}_{x \mu} \mathbf{W}_{m j}^{q} \mathbf{H}_{x \mu}^{T}\right) \mathbf{H}_{d}^{-1} .
\end{aligned}
$$

Finally, the parameters of $q_{X, \theta^{\prime}}\left(\mathbf{X}_{1: M}, \theta^{\prime}\right)$ are updated using (6), (11) and (13)

$$
\begin{array}{r}
\mathcal{N}\left(\left[\mathbf{X}_{1: M}^{T}, \mu_{1: K}^{T}\right]^{T} ;\left[\overline{\mathbf{X}}_{1: M}^{q, T}, \bar{\mu}_{1: M}^{q, T}\right]^{T}, \mathbf{W}^{\mathrm{q}}\right)= \\
\left(\prod_{m=1}^{M} \mathcal{N}\left(\mathbf{X}_{m} ; \overline{\mathbf{X}}_{m}^{0}, \mathbf{Q}_{m}\right)\right)\left(\prod_{j=1}^{K} \mathcal{N}\left(\mu_{j} ; \bar{\mu}_{j}^{0}, \mathbf{P}_{j}^{0}\right)\right) \\
\prod_{m} \prod_{i} \prod_{j} \mathcal{N}\left(\mathbf{y}_{m}^{i} ; \mathbf{H}_{x \mu}\left[\begin{array}{c}
\mathbf{X}_{m} \\
\mu_{j}
\end{array}\right]+\delta_{m}^{i j}, \overline{\mathbf{R}}\right)
\end{array}
$$

where $\overline{\mathbf{R}}=\mathbb{E}_{q_{\Sigma}}\{\tilde{\mathbf{R}}\}$, and

$$
\begin{aligned}
a_{j}^{q} & =a_{j}^{0}+\sum_{m} \sum_{i} \mathbb{E}_{q_{\mathbf{Z}}}\left\{z_{m}^{i j}\right\} \\
b_{j}^{q} & =b_{j}^{0}+\sum_{m} f\left(\mathbf{X}_{m}, \mu_{j}\right) \\
c^{q} & =c^{0}+\sum_{m} \sum_{i} \mathbb{E}_{q_{\mathbf{Z}}}\left\{z_{m}^{i 0}\right\} \\
e^{q} & =e^{0}+M .
\end{aligned}
$$




\section{Simulation And Results}

The presented algorithm is evaluated on a simulated scenario that is depicted in Figure 1. To evaluate the algorithm, two measures of performance have been used. The estimated map is compared to the true one using the integrated squared error (ISE) [18]. Using the ISE measure, all properties of the map, i.e., position of the landmarks, their weights and their extensions are accounted for in the evaluation. In addition, the estimated trajectory is evaluated using root mean square error (RMSE).

The following parameter values have been used in the simulations. The prior values for different parameters of the map are set to $\mathbf{S}_{j}^{0}=6 \mathbf{I}, \nu_{j}^{0}=8, a_{j}^{0}=0.1, b_{j}^{0}=0.2$, $c^{0}=0.05, e^{0}=0.1$. In addition it is assumed that a priori the map consists of 74 landmarks whose locations are uniformly distributed over the environment. The prior trajectory is generated using noisy odometry. The covariance of the measurement noise is set to $\mathbf{R}=\operatorname{diag}\left(\left[\sigma_{r}^{2}, \sigma_{\phi}^{2}\right]\right)$ where $\sigma_{r}=0.1 \mathrm{~m}$ and $\sigma_{\phi}=0.5^{\circ}$ and $\lambda_{c}=1$. The sensor's field of view has a range of $60 \mathrm{~m}$ and an angle of $\pm 30^{\circ}$.

The normalized ISE is depicted in Figure 2. As can be seen, the ISE improves from 0.94 to 0.29 . The RMSE of the estimated position of the vehicle is illustrated in Figure 3. This error is decreased from $0.43 \mathrm{~m}$ to 0.36 . Additionally, the estimated expected number of clutter measurements is 1.9 .

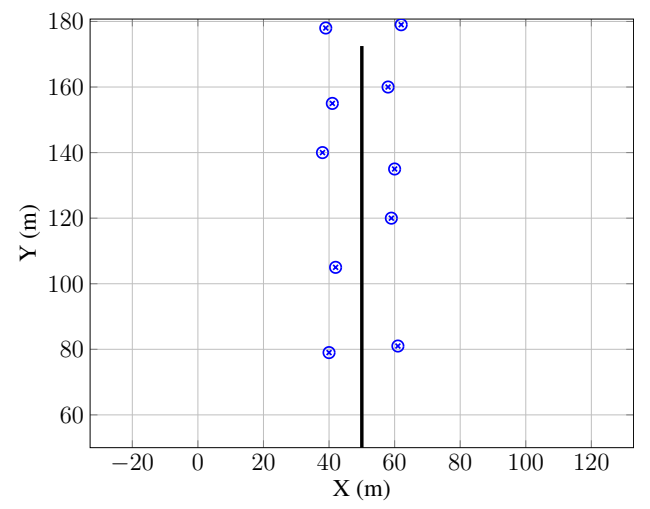

Figure 1. The scenario on which the algorithm is evaluated. The vehicle starts at position $(50,50)$. The extended landmarks are located by the road.

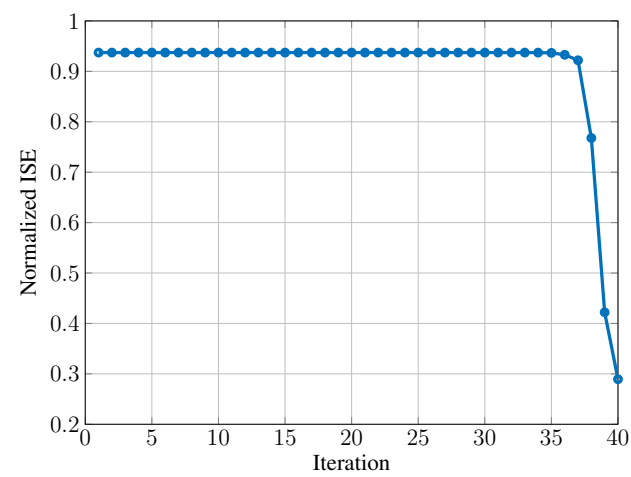

Figure 2. The normalized ISE of the estimated map.

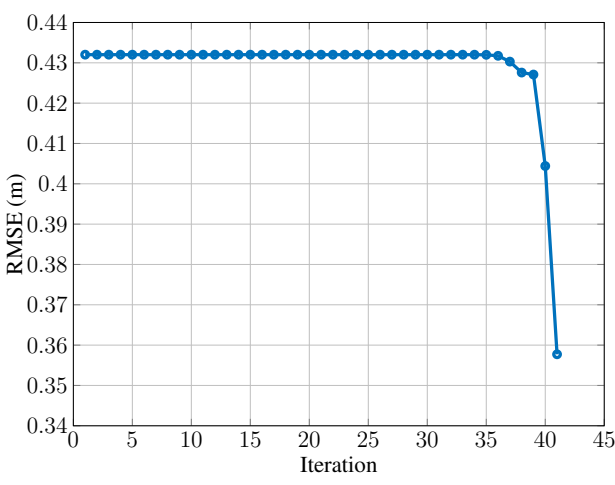

Figure 3. The RMSE of the estimated position.

\section{CONCLUSIONS}

In this paper, a radar-based SLAM solution using VBEM is presented. The algorithm is able to jointly estimate the data associations, the trajectory and the map by iteratively optimizing the KLD between the estimated and the exact posterior densities.

\section{REFERENCES}

[1] H. Durrant-Whyte and T. Bailey, "Simultaneous localization and mapping: part I," IEEE Robot. Autom. Mag., vol. 13, no. 2, 2006.

[2] T. Bailey and H. Durrant-Whyte, "Simultaneous localization and mapping (SLAM): Part ii," IEEE Robot. Autom. Mag., vol. 13, no. 3, 2006.

[3] J. Levinson et al., "Towards fully autonomous driving: Systems and algorithms," in Intelligent Vehicles Symp. (IV), 2011 IEEE, pp. 163-168.

[4] F. Moosmann and C. Stiller, "Velodyne SLAM," in Intelligent Vehicles Symp. (IV), 2011 IEEE, pp. 393-398.

[5] H. Lategahn, A. Geiger, and B. Kitt, "Visual SLAM for autonomous ground vehicles," in 2011 IEEE Int. Conf. Robotics and Automation.

[6] J. Ziegler et al., "Making Bertha drive? An autonomous journey on a historic route," IEEE Intell. Transp. Syst. Mag., vol. 6, no. 2, 2014.

[7] M. Adams, J. Mullane, and E. Jose, Robotic navigation and mapping with radar. Artech House, 2012.

[8] C. Lundquist, L. Hammarstrand, and F. Gustafsson, "Road intensity based mapping using radar measurements with a probability hypothesis density filter," IEEE Trans. Signal Process., vol. 59, no. 4, 2011.

[9] J. Mullane, B.-N. Vo, M. D. Adams, and B.-T. Vo, "A random-finite-set approach to Bayesian SLAM," IEEE Trans. Robot., vol. 27, no. 2, 2011.

[10] M. Adams, B.-N. Vo, R. Mahler, and J. Mullane, "SLAM gets a PHD: New concepts in map estimation," IEEE Robot. Autom. Mag., vol. 21, no. 2, pp. 26-37, 2014.

[11] K. Granström, C. Lundquist, and O. Orguner, "Extended target tracking using a Gaussian-mixture PHD filter," IEEE Trans. Aerosp. Electron. Syst., vol. 48, no. 4, pp. 3268-3286, 2012.

[12] J. W. Koch, "Bayesian approach to extended object and cluster tracking using random matrices," IEEE Trans. Aerosp. Electron. Syst., vol. 44, no. 3, pp. 1042-1059, 2008.

[13] M. Lundgren, "Bayesian filtering for automotive applications," Ph.D. dissertation, Chalmers University of Technology, 2015.

[14] A. P. Dempster, N. M. Laird, and D. B. Rubin, "Maximum likelihood from incomplete data via the EM algorithm," Journal of the royal statistical society. Series B (methodological), pp. 1-38, 1977.

[15] M. J. Beal, "Variational algorithms for approximate Bayesian inference," Ph.D. dissertation, University of London, 2003.

[16] S. Kullback and R. A. Leibler, "On information and sufficiency," Ann. Mathematical Statistics, pp. 79-86, 1951.

[17] Z. Ghahramani and M. J. Beal, "Propagation algorithms for variational Bayesian learning," Advances in neural information processing systems, pp. 507-513, 2001.

[18] J. L. Williams, "Gaussian mixture reduction for tracking multiple maneuvering targets in clutter," DTIC Document, Tech. Rep., 2003. 